\title{
Avaliação de Atributos de Textura de Núcleos Neoplásicos para a Classificação de Imagens Histológicas de Linfoma
}

\author{
Thaína A. A. Tosta ${ }^{1}$, Paulo R. de Faria ${ }^{2}$, Leandro A. Neves ${ }^{3}$, Marcelo Z. do Nascimento ${ }^{1,4}$ \\ ${ }^{1}$ Centro de Matemática, Computação e Cognição (CMCC) \\ Universidade Federal do ABC (UFABC) \\ CEP 09.210-580 - Santo André - SP \\ ${ }^{2}$ Departamento de Histologia e Morfologia, Instituto de Ciências Biomédicas \\ Universidade Federal de Uberlândia (UFU) \\ CEP 38.400-902 - Uberlândia - MG \\ ${ }^{3}$ Departamento de Ciências de Computação e Estatística \\ Universidade Estadual Paulista (UNESP) \\ CEP 15.054-000 - São José do Rio Preto - SP \\ ${ }^{4}$ Faculdade de Computação \\ Universidade Federal de Uberlândia (UFU) \\ CEP 38.408-100 - Uberlândia - MG \\ \{tosta.thaina, paulorfaria1976, neves.leandro,marcelo.zanchetta\}@gmail.com
}

\begin{abstract}
Through the use of image processing techniques, it is possible to develop computer-aided diagnosis systems so the analysis of histological samples can be more objective. Then, this paper presents an algorithm to classify histological images of follicular lymphoma and chronic lymphocytic leukemia. For identification of neoplastic nuclei, the $R$ channel extraction from RGB model was performed, followed by the application of histogram equalization, Gaussian filter, fuzzy 3-partition entropy with differential evolution, valley-emphasis and morphological operations. Texture features obtained through ranklet and wavelet transforms were evaluated using the classification by support vector machines. The segmentation of neoplastic nuclei of the considered lesions has reached an average of $80.49 \%$ of accuracy in comparison with the manual segmentation of a specialist. The classification of these images using the ranklet transform has reached $98.65 \%$ of accuracy, indicating the good performance of this technique for texture analysis of lymphoma images.
\end{abstract}

Resumo. Pela utilização de técnicas de processamento digital de imagens, é possível desenvolver sistemas de auxílio a diagnósticos para que a análise de amostras histológicas torne-se mais objetiva. Assim, este trabalho propõe um algoritmo para a classificação de imagens histológicas de linfoma folicular $e$ leucemia linfóide crônica. Para identificação de núcleos neoplásicos, o canal $R$ do modelo de cores RGB foi extraído, seguido pelas aplicações de equalização do histograma, filtro Gaussiano, fuzzy 3-partition entropy com o método de evolução diferencial, valley-emphasis e operações morfológicas. Atributos de textura obtidos pelas transformadas ranklet e wavelet foram avaliados pela classificação de máquinas de vetores suporte. A segmentação de núcleos neoplásicos das lesões proporcionou uma taxa média de acurácia de 80,49\% 
em relação à segmentação manual de um especialista. A classificação dessas imagens utilizando a transformada ranklet alcançou acurácia de 98,65\%, indicando o bom desempenho dessa técnica para a análise de textura de imagens de linfoma.

\section{Introdução}

A definição de diagnósticos precisos de câncer é essencial para a escolha de tratamentos eficientes para o paciente [Orlov et al. 2010]. No entanto, ao ser realizada manualmente por especialistas, essa tarefa torna-se subjetiva e dispendiosa [Sertel et al. 2010]. Por meio da digitalização de amostras histológicas coradas com hematoxilina-eosina (H\&E), métodos computacionais podem ser empregados para a identificação de regiões indicativas de diferentes tipos de câncer e para auxílio na definição de diagnósticos.

Os linfomas são neoplasias que se dividem em pelo menos 38 subtipos, de acordo com a Organização Mundial da Saúde. Seu desenvolvimento ocorre em células responsáveis pela defesa imunológica do organismo, chamadas linfócitos [Orlov et al. 2010]. Pela sua grande variedade de características histológicas e clínicas, os diagnósticos de seus subtipos tornam-se complexos. Essa condição também impõe limitações para aplicação de métodos de segmentação e classificação sobre imagens histológicas H\&E de linfoma.

Diferentes trabalhos da literatura propõem a classificação de imagens histológicas de linfoma para auxílio a seus diagnósticos. Esses trabalhos apresentam algoritmos para a classificação de imagens de Linfoma de Células do Manto (LCM), Linfoma Folicular (LF) e Leucemia Linfóide Crônica (LLC). Entre eles, [Tuzel et al. 2007] apresentou um método de classificação de linfomas e imagens de sangue com uma etapa de segmentação para identificação de núcleos e citoplasmas. Para a extração de características, filtros lineares foram aplicados e seus resultados foram agrupadas pelo K-means para a obtenção de histogramas de textons. A etapa de classificação foi realizada pela técnica de máquinas de vetores suporte (Support Vector Machines - SVM).

[Orlov et al. 2010] utilizou para a classificação de linfomas a avaliação de diferentes modelos de cores, métodos de redução de dimensionalidade e classificadores. Os atributos extraídos foram obtidos por transformadas de Fourier, Chebyshev, wavelet, Chebyshev de Fourier e wavelet de Fourier. A combinação entre a técnica de deconvolução, a função discriminante linear de Fisher e o classificador weighted neighbor distances alcançou os melhores resultados. [do Nascimento et al. 2015] apresentou um método de classificação de imagens de linfoma utilizando atributos de textura extraídos de transformadas wavelet estacionárias. Esses atributos foram reduzidos pelo método de análise de variância e classificados pelo SVM. [di Ruberto et al. 2015] apresentou um algoritmo para classificação de diferentes imagens histológicas, incluindo linfomas. Os modelos de cores RGB, HSV, L*a*b*, Luv e YCbCr foram avaliados para extração de características de textura. Pela análise de desempenho, o modelo HSV e matrizes de co-ocorrência alcançaram os melhores resultados utilizando o classificador SVM.

[Codella et al. 2016] também propôs a classificação de linfomas com uma etapa de pré-processamento com aumento de $20 \%$ de saturação de cores e equalização do histograma. Para identificar corantes de $\mathrm{H} \& \mathrm{E}$, e regiões celulares e não-celulares, diferentes processamentos foram realizados sobre os canais $R$ e $B$ do modelo RGB e pelo método 
de Otsu. A extração de atributos, como de canais de cores, arestas e wavelet, foi realizada considerando diferentes distribuições espaciais com classificação pelo SVM.

Uma das diferenças entre esses trabalhos é a utilização da etapa de segmentação para a identificação de regiões de interesse dessas lesões. Entre os estudos descritos, [Orlov et al. 2010], [do Nascimento et al. 2015] e [di Ruberto et al. 2015] não utilizaram essa etapa. Apesar dos bons resultados alcançados por essas propostas, a extração de atributos dessas regiões específicas podem contribuir para o desempenho da classificação [Orlov et al. 2010]. Além disso, trabalhos da literatura correlata não abordam análises de textura de núcleos neoplásicos para a classificação de imagens de LF e LLC, como proposto por este trabalho. Para a identificação dessas regiões de interesse, um método de limiarização foi aplicado por sua eficiência para a segmentação de estruturas histológicas [Oswal et al. 2013]. Nessa abordagem, um método de otimização evolutivo foi aplicado por sua robustez e bom desempenho em tempo de processamento [Sarkar et al. 2016].

Este trabalho propõe a avaliação de atributos de textura extraídos de núcleos celulares neoplásicos de imagens de LF e LLC para a classificação. Para isso, a identificação dessas regiões de interesse foi realizada pela combinação de técnicas divididas nas etapas de pré-processamento, segmentação e pós-processamento. Atributos de textura foram obtidos pelas transformadas ranklet e wavelet, e classificados pelo SVM, para definição da técnica mais adequada para auxílio a diagnósticos dessas lesões.

\section{Materiais e Métodos}

Nesta seção, são descritos o banco de imagens utilizado e o algoritmo proposto para classificação de imagens de LF e LLC.

\subsection{Base de Imagens}

As amostras histológicas utilizadas para validação do algoritmo proposto foram digitalizadas por meio de um microscópio de luz branca Zeiss Axioscope com lente objetiva de $20 \times$ e câmera colorida CCD AxioCam MR5. Tais amostras foram coradas com H\&E e constituem uma base de imagens pública, disponível em [Shamir et al. 2008]. Foram utilizadas 62 imagens de LF e 12 de LLC. Em ambas as classes, cada caso possui aproximadamente 2.000 células, quantidade próxima a outros trabalhos dedicados à segmentação de imagens histológicas e citológicas [Dimitropoulos et al. 2016, Wang et al. 2016].

\subsection{Método Proposto}

O método proposto utiliza cinco etapas em sua sequência metodológica: préprocessamento, segmentação, pós-processamento, extração de características e classificação. Para implementação do algoritmo proposto, foi utilizada a linguagem MATLAB $\cap$.

$\mathrm{Na}$ etapa de pré-processamento, as imagens foram inicialmente submetidas à extração do canal $R$ do modelo RGB, por seu maior contraste em relação ao fundo da imagem [Zorman et al. 2007]. As Figuras 1(b) e 2(b) ilustram esse processamento. Posteriormente, os métodos de equalização do histograma e filtro Gaussiano foram aplicados. A equalização do histograma foi utilizada para lidar com as diferenças de contraste e iluminação das imagens. O filtro Gaussiano permitiu a suavização da ima- 
gem por meio da convolução de uma máscara, de tamanho $3 \times 3$ pixels, com elementos definidos por uma função Gaussiana bidimensional, com atribuição de $\sigma$ ao valor 2 [Gonzalez and Woods 2000].

Para a segmentação dos núcleos neoplásicos, o método fuzzy 3partition [Yin et al. 2014] foi aplicado sobre o histograma normalizado das imagens pré-processadas. Esse método permitiu a definição de dois valores de limiar, obtendo três componentes identificados nas imagens: núcleos, citoplasmas e fundo da imagem. Para que essa definição fosse obtida, foram utilizados dois pares das funções $S$ e $Z$, representadas pelas Equações (1) e (2).

$$
\begin{gathered}
S(k, u, v, w)= \begin{cases}1, & k \leq u \\
1-\frac{(k-u)^{2}}{(w-u) \cdot(v-u)}, & u<k \leq v \\
\frac{(k-w)^{2}}{(w-u) \cdot(w-v)}, & v<k \leq w \\
0, & k>w\end{cases} \\
Z(k, u, v, w)=1-S(k, u, v, w) .
\end{gathered}
$$

Essas funções dividem os pixels em três fuzzy sets, definidos pelas Equações (3), (4) e (5), que representam os graus de associação a núcleo, citoplasma e fundo da imagem, respectivamente.

$$
\begin{gathered}
M_{\mathrm{n}}(k)=S\left(k, u_{1}, v_{1}, w_{1}\right), \\
M_{\mathrm{c}}(k)= \begin{cases}Z\left(k, u_{1}, v_{1}, w_{1}\right), & k \leq w_{1}, \\
S\left(k, u_{2}, v_{2}, w_{2}\right), & k>w_{1},\end{cases} \\
M_{\mathrm{f}}(k)=Z\left(k, u_{2}, v_{2}, w_{2}\right),
\end{gathered}
$$

onde, $u_{1}, v_{1}, w_{1}, u_{2}, v_{2}$ e $w_{2}$ determinam a distribuição dos graus de associação. Os valores de limiar são então definidos pelos pontos de intersecção entre $M_{\mathrm{n}}$ e $M_{\mathrm{c}}$, e $M_{\mathrm{c}}$ e $M_{\mathrm{f}}$.

Para a definição dos parâmetros $u_{1}, v_{1}, w_{1}, u_{2}, v_{2}$ e $w_{2}$, o método de Evolução Diferencial (ED) foi utilizado por sua simplicidade, robustez e convergência para ótimas soluções [Cuevas et al. 2016]. Essa técnica inicializa aleatoriamente sua população, neste trabalho, composta por 60 indivíduos representados por seis variáveis, correspondente à quantidade de parâmetros do método fuzzy 3-partition. A etapa seguinte consiste na definição da melhor solução proposta na população, $x_{\mathrm{r}_{1}}$, obtida pela função de avaliação, dada pela maximização da entropia de Shannon:

$$
H\left(u_{1}, v_{1}, w_{1}, u_{2}, v_{2}, w_{2}\right)=-P_{\mathrm{n}} \cdot \log \left(P_{\mathrm{n}}\right)-P_{\mathrm{c}} \cdot \log \left(P_{\mathrm{c}}\right)-P_{\mathrm{f}} \cdot \log \left(P_{\mathrm{f}}\right),
$$

onde, as probabilidades $P_{n}, P_{c}$ e $P_{f}$ representam as probabilidades de cada estrutura investigada: 


$$
P_{\mathrm{n}}=\sum_{k=0}^{255} h(k) \cdot M_{\mathrm{n}}(k), \quad P_{\mathrm{c}}=\sum_{k=0}^{255} h(k) \cdot M_{\mathrm{c}}(k), \quad P_{\mathrm{f}}=\sum_{k=0}^{255} h(k) \cdot M_{\mathrm{f}}(k),
$$

onde, $\mathrm{h}(\cdot)$ representa o histograma normalizado da imagem pré-processada.

Após essa definição, a solução $x_{\mathrm{r}_{1}}$ passa pela etapa de mutação considerando duas outras soluções da população $\left(x_{\mathrm{r}_{2}}\right.$ e $\left.x_{\mathrm{r}_{3}}\right)$, escolhidas aleatoriamente. Assim, é obtida uma nova solução $v_{\mathrm{i}}$ :

$$
v_{\mathrm{i}}=x_{\mathrm{r}_{1}}+F \cdot\left(x_{\mathrm{r}_{2}}-x_{\mathrm{r}_{3}}\right),
$$

onde, $i$ corresponde ao índice de um indivíduo da população e $F$ representa o fator de mutação, neste trabalho, atribuído ao valor 0,5.

Posteriormente, as soluções propostas na geração em execução terão seus parâmetros alterados considerando a condição representada por (9), levando à obtenção do chamado trial vector $\left(u_{\mathrm{i}}\right)$.

$$
u_{\mathrm{j}, \mathrm{i}}= \begin{cases}v_{\mathrm{j}, \mathrm{i}}, & \text { se } \operatorname{rand}(0,1) \leq C_{\mathrm{r}} \text { ou } j=j_{\mathrm{rand}}, \\ x_{\mathrm{j}, \mathrm{i}}, & \text { caso contrário, }\end{cases}
$$

onde, $j_{\text {rand }} \in 1,2, \ldots, 6$ e $0 \leq C_{\mathrm{r}} \leq 1$ representa a probabilidade de crossover, que controla a seleção de atributos a serem atribuídos à $u_{\mathrm{i}}$. Neste trabalho, essa variável assumiu o valor 0,2 .

Por fim, a operação de seleção é utilizada para otimização das soluções. Se o resultado da função de avaliação da solução $u_{\mathrm{i}}$ é maior ou igual ao da solução $x_{\mathrm{i}}, u_{\mathrm{i}}$ substitui $x_{\mathrm{i}}$ na geração posterior. O algoritmo é executado até que o critério de parada de [Hammouche et al. 2008] seja alcançado.

Na etapa de pós-processamento, pequenos ruídos com áreas menores que 10 pixels foram removidos. Além disso, a segmentação foi refinada pela remoção de regiões internucleares identificadas pelo método valley-emphasis [Ng 2006], aplicado sobre regiões com áreas maiores que 80 pixels. Nessa etapa, as operações morfológicas de abertura e dilatação [Gonzalez and Woods 2000] também foram utilizadas para que as regiões segmentadas tivessem seus atributos morfológicos próximos às marcações do especialista. Neste trabalho, os elementos estruturantes dessas operações possuíam distribuições em disco e quadrada, respectivamente.

Para extração de atributos de textura, foram avaliadas as medidas de entropia, energia, média e desvio padrão de componentes das transformadas wavelet coiflets 5 e ranklet. Transformadas wavelet descrevem de forma precisa atributos locais de sinais da imagem [Fiallos et al. 2015]. A decomposição de wavelets resultam na sub-banda de aproximação, que representa uma aproximação à imagem original, e em três sub-bandas que apresentam detalhes da imagem nas direções vertical $(V)$, horizontal $(H)$ e diagonal $(D)$ [Lahmiri 2016]. A primeira destaca a iluminação da imagem, e as sub-bandas restantes representam as intensidades das bordas de objetos [Abbas 2017]. 
Trasformadas wavelet discretas bidimensionais incluem quatro elementos críticos: uma função escalar bidimensional $\varphi(x, y)$ e três funções wavelet $\left(\psi^{V}(\mathrm{x}, \mathrm{y}), \psi^{H}(\mathrm{x}, \mathrm{y})\right.$, $\left.\psi^{D}(\mathrm{x}, \mathrm{y})\right)$. Essas funções são obtidas por duas funções unidimensionais. A transformada wavelet de uma imagem $f(x, y)$ de tamanho $M \times N$ é dada por [Yadav et al. 2015]:

$$
\begin{gathered}
\left.W_{\varphi}\left(j_{0}, r, c\right)=\frac{1}{\sqrt{M N}} \sum_{x=0}^{M-1} \sum_{y=0}^{N-1} f(x, y) \varphi_{j_{0}, r, c}(x, j)\right), \\
W_{\psi}^{i}(j, r, c)=\frac{1}{\sqrt{M N}} \sum_{x=0}^{M-1} \sum_{y=0}^{N-1} f(x, y) \psi_{j, r, c}^{i}(x, j), i=\{V, H, D\},
\end{gathered}
$$

onde, $j_{0}$ corresponde a uma escala inicial arbitrária. Uma aproximação da imagem $f(x, y)$ na escala $j_{0}$ é definida pelos coeficientes $W_{\varphi}\left(j_{0}, r, c\right)$. Detalhes verticais, horizontais e diagonais para a escala $j\left(j \geq j_{0}\right)$ são dados pelos coeficientes $W_{\psi}^{i}(j, r, c)$ [Yadav et al. 2015].

Uma das desvantagens das wavelets é a sua não robustez a transformações de brilho monotônicas, como obtidas pela equalização do histograma. Para a resolução dessa limitação, atributos estatísticos de classificação foram incorporados às wavelets, compondo as ranklets [Saha et al. 2011]. As transformadas ranklets são uma família de filtros multi-escalares com um padrão de orientação semelhante às wavelets de Haar [Smeraldi 2009]. Esse método é caracterizado por análises (1) de multi-resolução, (2) seletivas em orientação e (3) não paramétricas [Xi et al. 2017].

As análises de multi-resolução baseiam-se na definição de um conjunto de resoluções que determinam uma quantidade de regiões quadradas de cada imagem $I$. Em geral, para cada resolução, o número de regiões quadradas extraídas é dado por: $\left(I_{I}+1\right.$ $\left.I_{c}\right)^{2}$, onde $I_{I}$ representa o tamanho linear da imagem $I$ e $I_{c}$ corresponde ao tamanho linear da região a ser extraída. Para cada resolução, as análises seletivas em orientação dividem os $N$ pixels das regiões quadradas extraídas nos subconjuntos $T$ e $C$, definidos de acordo com as orientações vertical, horizontal e diagonal. Por fim, para cada orientação de cada resolução, as análises não paramétricas são realizadas pela classificação relativa de pixels, ao invés da utilização de seus valores de intensidade [Masotti and Campanini 2008]:

$$
R_{j}=\frac{\sum_{p \in T_{j}} \pi(p)-\frac{N}{4}\left(\frac{N}{2}+1\right)}{\frac{N^{2}}{8}}-1, j=V, H, D,
$$

onde, $\sum_{p \in T_{j}} \pi(p)$ é a soma das classificações dos pixels $\pi(p)$ em $T_{j}$. O coeficiente da derivada ranklet $R_{j}$ está mais próximo de +1 à medida que mais pixels de regiões em $T_{j}$ tenham valores de intensidade maiores que pixels em $C_{j}$. Consequentemente, quanto mais próximo de -1 , mais pixels de regiões em $C_{j}$ possuem maiores níveis de intensidade que pixels em $T_{j}$ [Masotti and Campanini 2008].

A classificação desses atributos foi realizada pelo SVM com a função de base radial e variável $\sigma$ atribuída ao valor 1. Para avaliação da classificação, o método de validação cruzada foi aplicado com divisão das amostras de dados em 10 partições. Para 
quantificação dos resultados, as medidas de acurácia, sensibilidade e especificidade foram utilizadas.

\section{Resultados e Discussão}

As Figuras 1 e 2 exemplificam resultados das etapas de pré-processamento, segmentação e pós-processamento de imagens H\&E de LF e LLC, respectivamente. As Figuras 1(f) e 2(f) apresentam as imagens originais com a identificação nuclear obtida pelo algoritmo proposto. As Figuras 1(g) e 2(g) correspondem às identificações nucleares realizadas manualmente pelo especialista. Pela análise qualitativa desses resultados, é possível observar algumas identificações errôneas, com regiões falso positivas e falso negativas. A métrica de acurácia para análise dessa etapa alcançou valores de 81,40\% e 79,59\% para as lesões LF e LLC, respectivamente, quantificando a taxa de concordância entre as segmentações.

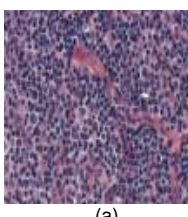

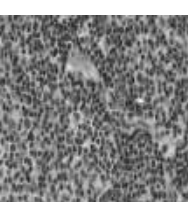

(b)

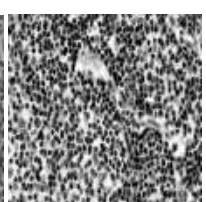

(c)

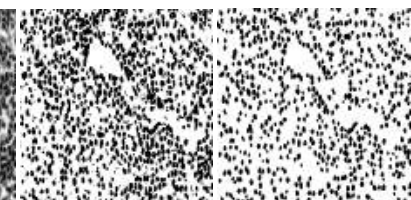

(d) (e)

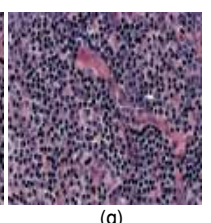

(g)

Figura 1. Exemplo de resultados obtidos para a segmentação de imagens de LF: (a) imagem original, (b) canal $R$ extraído, (c) resultado da etapa de préprocessamento, (d) regiões segmentadas pelo método ED, (e) imagem resultante do pós-processamento, (f) imagem original com a identificação das regiões segmentadas pelo método não supervisionado e $(\mathrm{g})$ segmentação manual realizada pelo especialista.
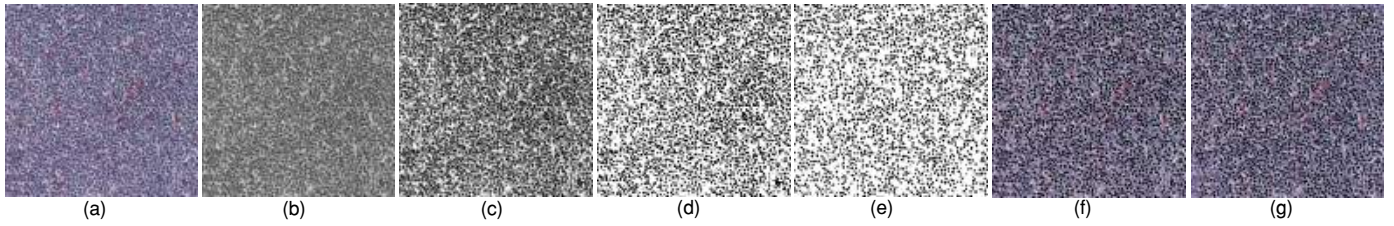

Figura 2. Segmentação de uma imagem de LLC pelo método proposto: (a) imagem original, (b) resultado da extração de seu canal $R$, (c) resultado do préprocessamento, (d) imagem resultante da segmentação, (e) imagem obtida pelo pós-processamento, (f) imagem original com a identificação das regiões segmentadas pelas etapas descritas e (g) identificações manuais do especialista.

A Tabela 1 apresenta os resultados da classificação $\mathrm{LF} \times$ LLC pelas medidas de acurácia, sensibilidade e especificidade utilizando os atributos de ranklets e wavelets. Pela variação dos atributos, os resultados de acurácia, sensibilidade e especificidade alcançaram diferenças de $14,87 \%, 14,52 \%$ e $16,66 \%$, respectivamente, onde os atributos ranklet apresentaram os maiores resultados em todas as métricas. Pela decomposição da imagem em seus componentes vertical, horizontal e diagonal, a ranklet é composta por coeficientes de medidas de regularidade da vizinhança dos pixels de cada componente [Masotti and Campanini 2008]. Por essa propriedade, os atributos de textura extraídos por essa técnica demonstraram robustez a variação de cores das imagens [Lo et al. 2015]. Os resultados obtidos indicam que os atributos extraídos por essa técnica oferecem informações robustas para a tarefa de diagnóstico de imagens de LF e LLC. 
Tabela 1. Resultados das medidas de acurácia, sensibilidade e especificidade da classificação $L F \times L L C$ pelos atributos ranklet e wavelet.

\begin{tabular}{|c|c|c|c|}
\hline Atributos & Acurácia & Sensibilidade & Especificidade \\
\hline Ranklet & $98,65 \%$ & $100 \%$ & $91,66 \%$ \\
\hline Wavelet & $83,78 \%$ & $85,48 \%$ & $75 \%$ \\
\hline
\end{tabular}

Tabela 2. Estudos de classificação de linfoma descritos por suas referências, número de classes investigadas e acurácias.

\begin{tabular}{|c|c|c|}
\hline Estudo de Classificação & $\mathbf{N}^{\mathbf{0}}$ de Classes & Acurácia \\
\hline [Codella et al. 2016] & 3 & $95,50 \%$ \\
\hline [Meng et al. 2010] & 3 & $92,70 \%$ \\
\hline [do Nascimento et al. 2015] & 3 & $100,00 \%$ \\
\hline [Orlov et al. 2010] & 3 & 99.00 \\
\hline Método Proposto & 2 & $98,65 \%$ \\
\hline
\end{tabular}

Para comparação com estudos que também propõem classificações de linfoma utilizando a mesma base de imagens desta proposta, a Tabela 2 apresenta seus resultados pela medida de acurácia. Entre os trabalhos apresentados, este estudo é o único que investiga a classificação de imagens de LF e LLC com a identificação de núcleos neoplásicos dessas lesões. Também é notório que os resultados obtidos por esta proposta são comparáveis aos estudos da literatura correlata.

\section{Conclusão}

Para a obtenção de diagnósticos de linfoma de forma objetiva, este trabalho propõe a classificação de imagens histológicas de linfoma. A combinação das técnicas empregadas no pré-processamento, segmentação e pós-processamento permitiram a identificação de núcleos neoplásicos com acurácias de 81,40\% e 79,59\% para as lesões LF e LLC, respectivamente, em relação à identificação de um especialista.

O desempenho da classificação das imagens, realizada pelo método SVM, foi analisado utilizando atributos de textura extraídos das transformadas ranklet e wavelet. Assim como para a representação de tumores [Angelini et al. 2006], a comparação entre atributos ranklet e wavelet permitiu definir a técnica ranklet como a mais adequada para a representação das imagens de linfoma analisadas.

Em trabalhos futuros, a etapa de segmentação será aprimorada para a remoção de regiões falso positivas e falso negativas. Ao solucionar essa limitação, espera-se que a classificação das imagens LF e LLC alcance resultados quantitativos máximos.

\section{Agradecimentos}

T.A.A.T. e M.Z.N. agradecem à CAPES e FAPEMIG (projeto TEC- APQ-02885-15) pelo suporte financeiro.

\section{Referências}

Abbas, Q. (2017). Segmentation of differential structures on computed tomography images for diagnosis lung-related diseases. Biomedical Signal Processing and Control, 33:325-334. 
Angelini, E., Campanini, R., Iampieri, E., Lanconelli, N., Masotti, M., Petkov, T., and Roffilli, M. (2006). A ranklet-based cad for digital mammography. In International Workshop on Digital Mammography, pages 340-346. Springer.

Codella, N., Moradi, M., Matasar, M., Syeda-Mahmood, T., and Smith, J. R. (2016). Lymphoma diagnosis in histopathology using a multi-stage visual learning approach. In SPIE Medical Imaging, pages 97910H-97910H. International Society for Optics and Photonics.

Cuevas, E., Zaldívar, D., and Perez-Cisneros, M. (2016). Image segmentation based on differential evolution optimization. In Applications of Evolutionary Computation in Image Processing and Pattern Recognition, pages 9-22. Springer.

di Ruberto, C., Fodde, G., and Putzu, L. (2015). On different colour spaces for medical colour image classification. In International Conference on Computer Analysis of Images and Patterns, pages 477-488. Springer.

Dimitropoulos, K., Barmpoutis, P., Koletsa, T., Kostopoulos, I., and Grammalidis, N. (2016). Automated detection and classification of nuclei in pax5 and h\&e-stained tissue sections of follicular lymphoma. Signal, Image and Video Processing, 11(1):145153.

do Nascimento, M. Z., Neves, L., Duarte, S. C., Duarte, Y. A. S., and Batista, V. R. (2015). Classification of histological images based on the stationary wavelet transform. In Journal of Physics: Conference Series, volume 574, page 012133. IOP Publishing.

Fiallos, C. B., Pérez, M. G., Conci, A., and Andaluz, V. H. (2015). Automatic detection of injuries in mammograms using image analysis techniques. In International Conference on Systems, Signals and Image Processing (IWSSIP), pages 245-248. IEEE.

Gonzalez, R. C. and Woods, R. E. (2000). Processamento de Imagens Digitais. Edgard Blucher.

Hammouche, K., Diaf, M., and Siarry, P. (2008). A multilevel automatic thresholding method based on a genetic algorithm for a fast image segmentation. Computer Vision and Image Understanding, 109(2):163-175.

Lahmiri, S. (2016). Image characterization by fractal descriptors in variational mode decomposition domain: Application to brain magnetic resonance. Physica A: Statistical Mechanics and its Applications, 456:235-243.

Lo, C. M., Chang, R. F., Huang, C. S., and Moon, W. K. (2015). Computer-aided diagnosis of breast tumors using textures from intensity transformed sonographic images. In Global Conference on Biomedical Engineering \& Asian-Pacific Conference on Medical and Biological Engineering, pages 124-127. Springer.

Masotti, M. and Campanini, R. (2008). Texture classification using invariant ranklet features. Pattern Recognition Letters, 29(14):1980-1986.

Meng, T., Lin, L., Shyu, M., and Chen, S. (2010). Histology image classification using supervised classification and multimodal fusion. In Multimedia (ISM), 2010 IEEE International Symposium on, pages 145-152. IEEE.

$\mathrm{Ng}, \mathrm{H}$. (2006). Automatic thresholding for defect detection. Pattern recognition letters, 27(14):1644-1649. 
Orlov, N. V., Chen, W. W., Eckley, D. M., Macura, T. J., Shamir, L., Jaffe, E. S., and Goldberg, I. G. (2010). Automatic classification of lymphoma images with transformbased global features. IEEE Transactions on Information Technology in Biomedicine, 14(4):1003-1013.

Oswal, V., Belle, A., Diegelmann, R., and Najarian, K. (2013). An entropy-based automated cell nuclei segmentation and quantification: Application in analysis of wound healing process. Computational and mathematical methods in medicine, 2013.

Saha, M., Chowdhury, S. R., and Roy, K. (2011). Ranklets: A qualitative review. In National Conference on Electronics, Communication and Signal Processing, pages $68-73$.

Sarkar, S., Das, S., and Chaudhuri, S. S. (2016). Hyper-spectral image ssegmentation using rényi entropy based multi-level thresholding aided with differential evolution. Expert Systems with Applications, 50:120-129.

Sertel, O., Lozanski, G., Shana'ah, A., and Gurcan, M. N. (2010). Computer-aided detection of centroblasts for follicular lymphoma grading using adaptive likelihood-based cell segmentation. IEEE Transactions on Biomedical Engineering, 57(10):2613-2616.

Shamir, L., Orlov, N., Eckley, D. M., Macura, T. J., and Goldberg, I. G. (2008). Iicbu 2008: A proposed benchmark suite for biological image analysis. Medical \& Biological Engineering \& Computing, 46(9):943-947.

Smeraldi, F. (2009). Fast algorithms for the computation of ranklets. In IEEE International Conference on Image Processing (ICIP), pages 3969-3972. IEEE.

Tuzel, O., Yang, L., Meer, P., and Foran, D. J. (2007). Classification of hematologic malignancies using texton signatures. Pattern Analysis and Applications, 10(4):277290.

Wang, P., Hu, X., Li, Y., Liu, Q., and Zhu, X. (2016). Automatic cell nuclei segmentation and classification of breast cancer histopathology images. Signal Processing, 122:113.

Xi, X., Xu, H., Shi, H., Zhang, C., Ding, H. Y., Zhang, G., Tang, Y., and Yin, Y. (2017). Robust texture analysis of multi-modal images using local structure preserving ranklet and multi-task learning for breast tumor diagnosis. Neurocomputing.

Yadav, A. R., Anand, R. S., Dewal, M. L., and Gupta, S. (2015). Performance analysis of discrete wavelet transform based first-order statistical texture features for hardwood species classification. In International Conference on Recent Trends in Computing, volume 57, pages 214-221. Elsevier.

Yin, S., Zhao, X., Wang, W., and Gong, M. (2014). Efficient multilevel image segmentation through fuzzy entropy maximization and graph cut optimization. Pattern Recognition, 47(9):2894-2907.

Zorman, M., Kokol, P., Lenic, M., de la Rosa, J. L. S., Sigut, J. F., and Alayon, S. (2007). Symbol-based machine learning approach for supervised segmentation of follicular lymphoma images. In IEEE International Symposium on Computer-Based Medical Systems, pages 115-120. IEEE. 